\title{
Los desastres y la Antropología: Entrevista a Rafael Mendoza
}

\author{
Alexia Potesta Cortez
}

Estudiante de Antropología de la Pontificia Universidad Católica del Perú (PUCP). e-mail: apotesta@pucp.edu.pe

\section{Dominique Barrueto Abanto}

Estudiante de Antropología de la Pontificia Universidad Católica del Perú (PUCP). e-mail:marie.barrueto@pucp.pe

\section{Bruno Ordoñez Hidalgo}

Estudiante de Antropología de la Pontificia Universidad Católica del Perú (PUCP). e-mail: a20150405@pucp.edu.pe

\section{Franco Villanueva Neme}

Estudiante de Antropología de la Pontificia Universidad Católica del Perú (PUCP). Miembro del Taller Museo Virtual Historia de la Escuela Peruana Centro de Investigaciones y Servicios Educativos (CISE) PUCP. e-mail: franco.villanueva@pucp.pe

\section{Resumen}

Esta entrevista tiene como objetivo acercarnos a los desastres desde la Antropología, a través de la perspectiva del doctor Rafael Mendoza (Ph.D en Antropología, University of Florida), especialista en el tema. Se aborda el concepto de desastre como un proceso de ruptura transformadora de la "normalidad", enmarcado en una determinada estructura social que condiciona este quiebre. El mismo concepto de "normalidad" es puesto en discusión como una categoría culturalmente relativa y constantemente redefinida por los actores sociales, y que ha cobrado un nuevo significado a partir de la pandemia de la COVID-19. Se profundiza en los límites conceptuales e implicancias del "desastre" y la "normalidad" desde la disciplina antropológica y los estudios cualitativos, desde un enfoque interdisciplinario. Asimismo, se intersectan las herramientas teóricas y metodológicas de la antropología de los desastres con la pandemia de la COVID-19, y se discute en qué medida se puede considerar un desastre, considerando las nociones de "nueva normalidad" de los actores sociales. La entrevista fue realizada de manera virtual, el viernes 22 de enero del 2020, en Lima y Quito simultáneamente.

\section{Palabras clave}

Desastres, Antropología, COVID-19, normalidad.

Nota editorial: el presente artículo fue recibido el 17-01-21 y aprobado el 08-02-21.

https://doi.org/10.18800/anthropia.2021.001 


\title{
Disasters and Anthropology: An Interview with Rafael Mendoza
}

\author{
Alexia Potesta Cortez \\ Anthropology student at the Pontifical Catholic University of Peru (PUCP). \\ e-mail: apotesta@pucp.edu.pe \\ Dominique Barrueto Abanto \\ Anthropology student at the Pontifical Catholic University of Peru (PUCP). \\ e-mail: marie.barrueto@pucp.pe
}

\section{Bruno Ordoñez Hidalgo}

Anthropology student at the Pontifical Catholic University of Peru (PUCP). e-mail: a20150405@pucp.edu.pe

\section{Franco Villanueva Neme}

Anthropology student at the Pontifical Catholic University of Peru (PUCP). Member of the Peruvian School History Virtual Museum Workshop - Research Center and Educational Services (CISE) PUCP.

e-mail: a20150405@pucp.edu.pe

\begin{abstract}
This interview aims to approach the topic of disasters from an anthropological perspective, with the viewpoint of a specialist on the topic, Dr. Rafael Mendoza (Ph.D in Anthropology, University of Florida). His approach to the concept of disaster views disaster as a process of rupture. Rupturing precisely what is deemed to be "normal" under certain social structures, therefore putting the very concept of "normal" into question, understanding it as a relative concept that is constantly being redefined by social actors, which has taken on a new meaning from the COVID-19 pandemic. It delves into the conceptual limits and implications of "disaster" and "normal" from an interdisciplinary approach which encompasses the anthropological discipline and qualitative studies. Likewise, the theoretical and methodological tools of the anthropology of disasters intersect with the COVID-19 pandemic. Therefore, there is a discussion about to what extent can a disaster be considered a disaster, considering the notions of a "new normal" of social actors. The interview was conducted virtually on Friday, January 22, 2020, in Lima and Quito simultaneously.
\end{abstract}

\section{Keywords}

Disasters, Anthropology, COVID-19, normality. 


\section{Introducción}

Rafael Mendoza (PhD en Antropología, University of Florida) es, actualmente, un reconocido consultor y profesor en el área de la Antropología de los desastres. Asimismo, tiene una larga trayectoria en temas sobre Antropología ambiental, cambio climático, territorios indígenas, conservación y Amazonía. Realizó el pregrado en Antropología en la Pontificia Universidad Católica del Perú y sus estudios de maestría en la FLACSO de Ecuador en Estudios Socio Ambientales. Tiene alrededor de quince años de experiencia en trabajos de conservación y desarrollo en la región andino amazónica, en organizaciones indígenas, ONGs de conservación y consultorías para el Estado; como también, ha apoyado en la consolidación de los territorios indígenas y sus estrategias de desarrollo local. Ha sido profesor hasta el 2019 en The School For Field Studies (SFS) en su sede de Iquitos y en la Universidad Católica del Perú (PUCP). Recientemente, ha realizado trabajos para WWF, el Ministerio de Cultura del Perú y la Coordinadora de Organizaciones Indígenas de la Cuenca Amazónica (COICA).

Esta breve entrevista tiene como propósito brindar a los lectores $\mathrm{y}$ lectoras un panorama general de la Antropología de los desastres, temática

Un desastre es un evento de quiebre de un contexto que las personas consideran normal. [...] Implica que la gente sienta que existe una anormalidad, un periodo de vacío en el cual no sabes a dónde vas a ir, de incertidumbre y caos.

del presente Dossier. Como los artículos que forman parte del dossier tocan temas más específicos, desde Anthropía vimos necesario un espacio en el que se aborde el tema de forma amplia. Consideramos importante para ese acercamiento utilizar el formato de una entrevista con un especialista en el tema como ideal para despertar el interés en este y que a la vez al leerse pueda transmitir mucha más cercanía, ya que sigue una línea de conversación. Los temas abordados serán los desastres, como concepto en las Ciencias Sociales, los acercamientos metodológicos y marcos conceptuales relacionados a esta rama, sus etapas y actores, y la forma como puede vincularse con la pandemia de la COVID-19.

\section{¿Cómo podemos entender un desastre y cuáles son sus límites conceptuales?}

Un desastre es un evento de quiebre de un contexto que las personas consideran normal. No ocurre dentro de un vacío; ocurre dentro de una estructura social, dentro de un tiempo y un espacio concretos, e implica que la gente sienta que existe una anormalidad, un periodo de vacío en el cual no sabes a dónde vas a ir, de incertidumbre y caos. Este evento catastrófico se vive como una ruptura social. Puede producir muchas muertes y puede destruir las instituciones sociales. En este sentido, desde una perspectiva histórica, solo puede haber tenido este efecto destructor porque existe una estructura social previa que crea las condiciones para 
que el desastre ocurra. ¿Qué cosa puede ser denominada un desastre? En gran parte, es nuestra propia decisión: nosotros podemos denominar un desastre a eventos que nos parecen que trastocan o destruyen la normalidad; sin embargo, hay demasiados eventos que caen dentro de este marco de lo que es catastrófico.

La gente puede considerar que una huelga de buses es un evento catastrófico para la economía nacional. La gente también puede considerar que la pérdida contra Chile en las eliminatorias de 1998, cuando no clasificamos al Mundial, es un desastre para mucha gente; ya que nuestra identidad estuvo seriamente dañada. Al respecto, me acuerdo que la población se sentía pésima y estaba en la calle llorando porque estábamos tan cerca de ir al Mundial luego de 15 años y al final no se logró. No obstante, en términos conceptuales y de la antropología del desastre, estos eventos no pueden ser un desastre porque no han sido destruidas las instituciones sociales; asimismo, no ha habido una gran pérdida de vidas, la normalidad no ha cambiado, no ha habido una destrucción de la infraestructura o la cotidianidad, y eso es lo que marca un desastre.

\section{¿Cuál cree que sea el aporte de la Antropología, como disciplina, al estudio de los desastres?}

Hay una serie de aspectos que surgen después de un desastre y han sido estudiados privilegiadamente por la Antropología: el daño a la identidad, la recuperación, la resiliencia, cómo las personas pueden afrontar el desastre, la pérdida de vidas, la destrucción de todo el entorno o gran parte del entorno, la desaparición física de gente amada y al mismo tiempo empezar a recuperarse con relativa rapidez; estos sentimientos de solidaridad colectiva, de comunidad. Los desastres se estudian desde hace 150 años, desde que existen las Ciencias Sociales. Desde un enfoque académico y formal se han estudiado desastres naturales, guerras, migraciones, entre otros. Sin embargo, se han tendido a analizar originalmente desde un punto de vista cuantitativo, en términos de números, de abstracciones sociales, de pérdidas de vida o de pérdidas económicas. La Antropología, por el contrario, es la Ciencia Social que ha proporcionado una mirada cualitativa y desde adentro.

\section{A partir de las vivencias o de las diferentes experiencias que pueden tener las personas, ¿qué marcos teóricos o qué metodologías serían útiles para abordar los desastres desde la Antropología?}

Yo no creo que pueda haber un método o una teoría única con la que se pueda abordar un desastre. En la mayoría de las veces, escogerlos es contextual y, generalmente, los antropólogos ya usamos una miscelánea de enfoques cuando tratamos un evento. Ya no podemos ser solo marxistas, ya no podemos ser estructuralistas completamente, estructural-funcionalistas, interpretativos, no. El enfoque siempre es holístico y de acuerdo al evento se plantea cuál es el más relevante. Además, escogemos el enfoque que puede ayudarnos a conseguir mayor información o el que tiene una mirada más incisiva con el fenómeno. Entonces, no hay una aproximación privilegiada para los desastres. 
Ahora, uno puede ver que dentro de los desastres hay patrones. Primero, tienes el periodo de lo que se llama normalidad. Segundo, tienes el evento. Como ya he mencionado, dicho evento causa un trastorno social que ocurre a raíz de la ruptura de las instituciones, ruptura del espacio y tiempo, y la pérdida de vidas. Durante este segundo período, ocurre un momento de solidaridad comunitaria; surge la necesidad de apoyarse; es decir, el espíritu colectivo que es bien humano para salvarse entre sí y a los demás. En momentos de desesperación, la población muestra su lado más humano y solidario; fenómeno que no suele durar mucho, generalmente solo unos días. Luego, viene el periodo de la normalización o el periodo post-desastre. Llega la ayuda internacional y empiezan los conflictos: se revelan rivalidades y demás tensiones sociales. Surgen las cuestiones de quiénes son los que vienen a ayudarnos y cuál es el rol del Estado. Emerge un conflicto también con la identidad. Según la teoría del don de Marcel Mauss, los regalos se tienen que retribuir de alguna forma. Esa retribución es lo que permite que una identidad sea sana y fuerte. En el momento que no se pueda retribuir, la identidad comienza a dañarse porque ya no se es un ser humano completo, ya no se está devolviendo. Las identidades, después de un desastre, pueden ser golpeadas porque la gente recibe ayuda pero no pueden devolverla. En este sentido, tenemos patrones y secuencias de los eventos que se pueden emplear, para su estudio, según el caso específico del desastre.

Dicho lo anterior, ¿Considera que el estudio de los desastres, desde la disciplina antropológica, requiere de un acercamiento multidisciplinario?

Sí, obviamente. Una persona no alcanza a tener todas las comprensiones de otras ramas. Por lo general, los antropólogos no saben de economía, entonces no saben las implicancias económicas de los eventos. Los sociólogos piensan parecido a los antropólogos, pero tienen otras tradiciones, sus propios padres fundadores. Los geógrafos piensan bastante en el espacio, en el significado de este. Los psicólogos sociales hacen cosas más aplicadas, ellos estudian, por ejemplo, cómo la gente reacciona durante un desastre. Los antropólogos, generalmente, estudiamos lo que pasa, o el proceso que hay, después de un desastre. Hacemos el estudio trabajo de campo y tenemos sensibilidad cualitativa, pero no solemos estudiar lo que ocurre en ese momento, en esas primeras horas del desastre. En cambio, los psicólogos sociales sí estudian el inicio del fenómeno y están preocupados en observar la reacción social en ese momento. Entonces, siempre es necesaria la mirada multidisciplinaria. Cuantas más miradas tengas, y desde diferentes ángulos, se enriquece el estudio de un evento.

\section{Ahora bien, los desastres suelen ser automáticamente correlacionados a la acción de la naturaleza, así se soslaya la injerencia de la acción humana. Frente a ello, ¿de qué forma cree que se puede problematizar la dicotomía naturaleza/cultura desde el concepto de desastre?}

Lo que usualmente se dice en los estudios de desastres es que, si ocurre un terremoto en una isla vacía e inhabitada de 9 puntos en la escala de Richter, no es un desastre, 
es solamente un terremoto en una isla. Si hay humanos, pero no les pasa nada porque han estado distribuidos y organizados de tal forma de que este terremoto no cause daños a la sociedad que vive ahí, tampoco es considerado un desastre. Pero si a esta presencia humana le ocurre una pérdida significativa de vidas y las instituciones son destruidas, entonces sí es un desastre. En este sentido, la pregunta típica es: ¿hasta qué punto un evento que es natural puede ser considerado un desastre si no es por la mirada humana? Es nuestra mirada lo que hace que fuerzas naturales, fuerzas que están más allá de los humanos, sean consideradas desastres o no sean consideradas desastres. Es nuestra mirada la que clasifica el mundo. Entonces, ¿hasta qué punto podemos dividir el entorno si nosotros sabemos que esa clasificación solamente es hecha por nosotros, por nuestra mirada y en función de nuestros propios intereses, ideologías, y nuestro propio medio de vida?

El caso típico para tratar la dicotomía naturaleza / cultura es el de los bosques amazónicos. Esto no es Antropología de los desastres, es Antropología amazónica. Los bosques amazónicos, tradicionalmente, se han visto como naturales, "son totalmente naturales", "los bosques han evolucionado por sí mismos". Bueno, antropólogos, etnobotánicos y arqueólogos han mostrado que la composición de los bosques amazónicos son producto de 6.000 años de injerencia humana. Los humanos han ido extirpando las especies que no

¿Hasta qué punto un evento que es natural puede ser considerado un desastre si no es por la mirada humana? Es nuestra mirada lo que hace que fuerzas naturales, fuerzas que están más allá de los humanos, sean consideradas desastres 0 no sean consideradas desastres. les eran útiles en el bosque y luego las han reemplazado por especies que creían que sí lo eran. Entonces, si tengo palmeras que dan frutos que a mí me gustan, agarro las semillas de la fruta y empiezo a repartirlas y tirarlas por el bosque. Si existe una planta que es venenosa, ¿Qué es lo que hago? La rozo y la mato, busco eliminar esas plantas que son potencialmente venenosas para nosotros. Si existen

lianas de palmeras que son útiles para construir nuestras casas, nuestras malocas o para hacer las redes de pescar. ¿Qué hago? Promuevo que haya más de esas palmeras en el bosque. Si haces la suma acumulada de 6.000 años de actividad humana sobre un bosque, resulta que la composición del bosque ha sido, en gran parte, modificada por la actividad humana.

Entonces, ¿en qué medida ese bosque es natural? o ¿en qué medida ese bosque es antropogénico, creado por nosotros? De igual manera, cada planta tiene su propio ritmo de crecimiento y su propia distribución que es natural de su especie. Sin embargo, nosotros nos hemos involucrado en todo eso. La separación entre naturaleza y cultura es muy difícil de desentrañar. No solamente es muy difícil, ¿es práctico hacerlo? La pregunta sería ¿para qué crear dos categorías, naturaleza y cultura, cuando sabes que esta clasificación como naturaleza es una construcción que te permite legitimar algo? ¿Para qué hacer esa diferencia? 


\section{La pandemia por la COVID-19 ha tenido una influencia devastadora y transformadora en los seres humanos, así como en nuestras instituciones sociales y sanitarias. Ante ello, ¿cómo podemos comprender esta crisis sanitaria desde la Antropología de los desastres?}

Originalmente, cuando empezó la pandemia y hubo cuarentena, el análisis era secuencial, encajaba bien con la Antropología de los desastres. Cuando empieza la emergencia por COVID, los países no estaban preparados para el evento: algunos, por una estructura social y por un Estado que había enfatizado más en salud, estaban mejor preparados, en teoría; mientras que otros países, como el Perú, donde la inversión pública en salud siempre ha sido reducida y grandes extensiones del país han sido abandonadas, nunca fueron tratadas ni incluidas dentro del crecimiento del Estado-nación, iban a ser los más impactados por la COVID-19. Sabemos que el Perú es uno de los países más impactados por la COVID.

El marco de la antropología del desastre encaja. Hubo un período que la gente consideraba de normalidad y tienes la llegada inesperada de un evento. Existió un espacio en el cual la gente empieza a sentir la solidaridad. No sé si ustedes veían en sus barrios, pero recuerdo que a las 8 de la noche la gente salía a aplaudir a los policías y gritaban "Contigo Perú", "Te amo Perú". Claro, había ese sentimiento, al menos por las primeras semanas. Entonces, eso coincide con la Antropología de los desastres. Es decir, tienes toda la secuencia: normalidad, ruptura, solidaridad y, después de la solidaridad, viene el proceso de interrogarse qué es lo que pasó y por qué nos está pasando, por qué a nosotros nos pega más fuerte que a otros. Entonces, empiezas a replantearte qué es lo que hicimos mal, en qué fallaron nuestras instituciones. Hasta ahí todo encaja dentro de la secuencia de los desastres. Es decir, la duración de la pandemia y el concepto de 'nueva normalidad' post desastre ha evidenciado que realmente estos procesos encajan dentro de los patrones comunes de la teoría de desastres, tomando en cuenta que el desastre en este caso aún continúa.

\section{A propósito de esta coyuntura de la COVID-19, desde una perspectiva antropológica, ¿qué concebimos, entonces, por "normalidad"?}

Desde la perspectiva antropológica, "normalidad" aplica a lo que la gente cree que es normal. Esta pregunta no me la han hecho antes y no creo que la haya leído antes, así que estamos desarrollando teoría. Yo diría, basándonos en enfoques antropológicos como el relativismo cultural y en cuáles son las percepciones y prácticas de las personas, la normalidad se refiere a cómo funciona una sociedad y los patrones por los cuales funciona la sociedad, que además son patrones temporales. Por ejemplo, la gente se levanta alrededor de las 6 , mandan a sus hijos al colegio a las 8 de la mañana, salen a las 2 de la tarde, van en bus, con movilidad particular o caminando, la gente toma el bus va al trabajo, regresa a las 5 de la tarde, o a las 6 de la tarde, socializan en un bar. En todo ese tipo de cosas hay una estructura social y material que apoya este funcionamiento de la sociedad, una serie de relaciones sociales, un juego cultural y 
simbólico que mantiene y reproduce estas relaciones materiales y sociales dentro de una comunidad. Las mismas personas perciben y categorizan aquello como lo normal. Igualmente, los antropólogos categorizamos eso como normal en la media que para la gente lo sea; entonces, se estudia como una categoría emic, como normal.

En esta línea, la nueva normalidad o ruptura de la normalidad es la fragmentación de este espacio social y material en la cual funciona la sociedad, en la que las personas se despiertan a las 6 de la mañana, preparan su desayuno, mandan a sus hijos físicamente al colegio, se van físicamente al trabajo, al colegio, socializan después del trabajo en un bar o en un parque. La ruptura de ello se refleja en que las personas ya no se levantan a las 6; de repente han cambiados sus horarios; tal vez los hijos ya no van al colegio y se quedan en casa; ya no van al trabajo y lo hacen desde casa; socializan de otra forma y usan los medios visuales para ver a sus amigos; tienen que usar una mascarilla para salir a la calle; se rigen por un horario para salir a la calle. Todo ello está predefinido y las personas lo sienten como una ruptura, y se siente y se define por la misma gente como una nueva normalidad. Como antropólogo, si la gente lo define así, yo estudio el concepto y lo asumo como nueva normalidad, y lo acepto. No tengo los elementos para juzgar si eso es normal o anormal, la variedad de condiciones humanas y la vida humana es tan rica que es muy difícil definir lo que es normal o anormal. La experiencia de relaciones humanas y rupturas materiales es tan grande en nuestra historia, diferencias materiales, de socialización, diferencias culturales es tan grande que es difícil hablar de lo que es normal o anormal.

Antes de la pandemia no había escuchado usar la palabra normalidad como una categoría. Existía la categoría pero creo que no le habíamos prestado atención; al menos en la antropología del desastre la pensábamos como una ruptura temporal breve. No sé si la gente hablaba de nueva normalidad, por ejemplo, después del terremoto de Yungay, pasan varios años para reconstruir Yungay, pero no se si gente usaba esa palabra de nueva normalidad para referirse al Yungay antiguo y al moderno. En cambio, ahora sí usamos el término. Creo que se están abriendo nuevos campos y nuevos significados y somos conscientes de eso.

Pero, también, así tal como existe el discurso en el contexto de la pandemia acerca de la nueva normalidad, no tenemos un referente similar. Entonces, los patrones al inicio de la pandemia son similares a las de un desastre: normalidad, ruptura, solidaridad, replanteamiento de lo que ha pasado; se abren heridas, hay conflicto y reconstrucción. Bueno, en la pandemia actual alrededor del mundo no hay todavía esta última parte, la reconstrucción. ¿Cuál es la normalidad? No la sabemos aún. ¿Va a haber una nueva normalidad o vamos a volver a la antigua normalidad? No lo sabemos. ¿Qué cambios va a haber en la economía mundial? ¿Qué cambios va a haber en las instituciones estatales? Tampoco lo sabemos. Supondríamos que los Estados van a invertir más en salud, pero en verdad ni esa certeza tenemos. Se puede suponer que la COVID va a terminar. Es decir, la mirada optimista es que con las vacunas, la COVID, va a desaparecer o va a simplemente convertirse en 
una gripe normal, común y corriente para todos nosotros para al final de este año. Pero tampoco lo sabemos porque también lo más pesimistas de nosotros piensan que pueden aparecer cepas, variantes que alarguen el proceso, que alarguen la COVID por un sinnúmero de años más.

Ya llevamos casi un año de la pandemia por COVID-19 ¿Hasta qué medida podemos conceptualizar esto como un desastre si consideramos que la supuesta normalidad que fue rota hace un año se está convirtiendo en la nueva normalidad?

Siempre va a haber una nueva normalidad, estamos jugando con términos. Después de que haya un desastre, por ejemplo, un terremoto, la ciudad se reconstruye, habrá gente que podría considerar que hay una nueva normalidad, y gente que dirá que hemos regresado a la normalidad, al estado idílico pre terremoto, pero las condiciones siempre cambian. La normalidad es un concepto, pero eso no impide que todo esté cambiando. Como dijo Heráclito, nadie se baña en el mismo río dos veces, porque el tiempo pasa y las condiciones materiales van transformándose permanentemente. Lo que pasa es que en eventos como la pandemia o un terremoto cambian las condiciones materiales y sociales abruptamente, de un modo que no se hace en un tiempo lineal. O sea, las sociedades siempre se transforman, pero muchas veces no nos damos cuenta de que se están transformando. No se tiene una autoconsciencia permanente de que se está transformando. Todo va cambiando, pero no siempre tiene importancia porque a veces el cambio es insignificante. No se conceptualiza.

La pandemia sí cumple con una serie de condiciones, un impacto demográfico, un número de muertos, hay cambios en el uso del espacio y

Siempre va a haber una nueva normalidad. [...] Las condiciones siempre cambian. La normalidad es un concepto, pero eso no impide que todo esté cambiando. como dijo Heráclito, nadie se baña en el mismo río dos veces, porque el tiempo pasa y las condiciones materiales van transformándose permanentemente.

el tiempo, hay cambios en las relaciones materiales, hay cambios en las relaciones sociales y hay esta sensación de que hay una ruptura del tiempo. Todo esto se cumple en la pandemia, dura un largo tiempo, pero tampoco está establecido que un desastre no puede durar mucho tiempo. En Leningrado, los soviéticos resistieron durante tres años y fue un desastre para la población, marcó un antes y un después. No hay nada escrito que diga que un desastre tenga que durar sólo cinco minutos y que después venga la reconstrucción. ¿El tiempo post COVID, si es que tenemos una vacuna que elimina los peligros de la COVID-19, va a ser un regreso a la normalidad pasada? Objetivamente no, pero la gente lo podría percibir como que sí. Objetivamente, no, porque ya hubo pérdidas materiales y sobre eso se han construido nuevas relaciones, una nueva realidad. Pero la gente podría percibir que 
hemos regresado. O podría decir que es una normalidad que se parece a la anterior, pero no es la misma. Entonces, hay que tener cuidado porque estamos jugando con cosas que son categorías o conceptos bien abstractos. Por lo tanto, es difícil leer o entender la COVID-19, cuando todavía estamos en el proceso, cuando todavía no se ha acabado y ni siquiera sabemos cuándo va a acabar porque no sabemos realmente si acaba este año o ya va a ser parte de la cotidianidad humana.

\section{Muchos académicos y académicas, de Ciencias Sociales, se están acercando a la Antropología de los desastres a partir de la pandemia por la COVID-19. Frente a ello, ¿cómo percibe el panorama de investigación en este campo de estudio en el futuro próximo?}

En principio, yo creo que debería haber mayor inversión en entender estos eventos. Entonces, si hubiera más antropólogos que trabajaran Antropología de los desastres, si desde el Estado o desde las instituciones del Estado hubiera un interés en entender cómo se puede prevenir un potencial desastre, cómo se puede responder al mismo y cómo se puede reconstruir, sería grandioso. Debería haber mayor inversión y más gente involucrada en lo que se llama Antropología de los desastres; y son muy pocos, no solamente en el Perú, sino en el mundo. Ahora, la cosa también es que no solamente lo debemos ver como una cosa exclusiva de los antropólogos, porque los sociólogos, geógrafos y economistas también deberían trabajar eso. De hecho, yo esperaría que el dossier pueda ser también ampliado o difundido entre sectores que no son solamente antropólogos, sino en otros lados, porque es algo que nos concierne a todos. Los peruanos sufrimos, pues, desastres tras desastres.

\section{Reflexiones finales}

Con todo lo mencionado, se evidencia que los desastres son eventos de urgente análisis desde las Ciencias Sociales. Constituyen momentos de desestructuración, crisis y reconstrucción de las relaciones y estructuras sociales, además de una sensación de ruptura con la normalidad. El carácter multidisciplinario de la Antropología de los desastres nos brinda un enfoque que complementa nuestro bagaje académico para analizar cómo las sociedades afrontan situaciones de crisis y las repercusiones de un desastre como la pandemia de la COVID-19 en las estructuras sociales y en la noción de "normalidad". De esta forma podemos generar un juicio crítico frente a la manera desigual en que los desastres afectan a los estratos sociales más vulnerables. Actualmente, nos encontramos en un escenario de incertidumbre frente a los cambios, que para la Antropología del Desastre forman parte del ciclo postdesastre. Ello nos acerca a reflexionar sobre el carácter cambiante de las condiciones que percibimos como "normales" y lo expuesta que está la sociedad ante eventos transformadores y de quiebre. 